\title{
Low Volume Polyethylene Glycol (PEG) Plus Ascorbic Acid, a Valid Alternative to Standard PEG
}

\author{
Su Hwan Kim and Ji Won Kim \\ Department of Internal Medicine, Seoul National University Boramae Medical Center, Seoul National University College of Medicine, Seoul, \\ Korea
}

See "Does Polyethylene Glycol (PEG) Plus Ascorbic Acid Induce More Mucosal Injuries than Split-Dose 4-L PEG during Bowel Preparation?" by Min Sung Kim, et al. on page 237-243, Vol. 10. No. 2, 2016

Adequate bowel preparation is essential to improve colonoscopy quality. ${ }^{1}$ Inadequate bowel preparation may result in lower colonoscopy completion rate, longer duration of colonoscopy, and lower diagnostic yield for polyps. ${ }^{2}$ Polyethylene glycol (PEG) has been shown to be safe and effective, and thus regarded as the gold standard for bowel preparation before colonoscopy. ${ }^{3}$ However, the standard PEG regimen may result in reduced tolerability and poor compliance due to its high volume. Sodium phosphate $(\mathrm{NaP})$ and sodium picosulfate (Pico) regimens were developed as an effort to reduce patient discomfort, and have shown better compliance and similar bowel cleansing efficacy compared with standard PEG. ${ }^{4,5}$ However, their use has been limited because they induced mucosal inflammation 10-fold more frequently than PEG. ${ }^{6}$ Recent studies comparing 2-L PEG plus ascorbic acid (2-L PEG+Asc) and 4-L PEG reported that 2-L PEG+Asc was equally as efficacious as 4-L PEG. ${ }^{7}$ Excessive ascorbic acid cannot be absorbed in bowel lumen and can act as an osmotic laxative. In this respect, concerns can be raised about mucosal inflammations induced by 2-L PEG+Asc.

In this randomized and investigator-blinded study, Kim et al. compared the rate of mucosal injury, efficacy, and patient affinity for the preparation between 4-L PEG and 2-L PEG+Asc in consecutive outpatients. ${ }^{8}$ With regard to mucosal inflammation, there was no significant difference between the two groups (4-L PEG vs 2-L PEG+Asc, 3.1\% vs 3.7\%). The total score of the Ottawa bowel preparation scale was not significantly different between the two groups $(4.19 \pm 2.26$ vs $4.41 \pm 2.07, p=0.376)$. Patient compliance showed no significant difference between the two groups $(96.3 \%$ vs $96.9 \%, p=0.768)$. Better patient pref- erence was shown in the 2-L PEG+Asc group (35.6\% vs 64.6\%, $\mathrm{p}=0.001$ ).

This study has several implications. First, authors indicated that acute mucosal inflammation did not occur significantly more with 2-L PEG+Asc compared to 4-L PEG. This result is meaningful by itself because it indicated that we now have a bowel preparation regimen that has lower volume than 4-L PEG and no concern for acute mucosal inflammation complicating the diagnosis of patients with inflammatory bowel diseases (IBD) or taking nonsteroidal anti-inflammatory drugs (NSAIDs). No significant difference in the rate of acute mucosal inflammation and adverse events could be explained by the fact that patients taking 2-L+Asc regimen were still required to ingest additional 1-L of clear liquids, even though the total amount of fluids they ingested was less than 4-L PEG. Second, split dose regimen of PEG, which is currently considered better than nonsplit dose regimen in bowel preparation efficacy, was applied to both groups in this study. ${ }^{8}$ Thus, the results of this study might give us more useful information than previous studies with nonsplit dose regimen.

Despite the positive implications of this study, some issues need to be considered. First, duration of the interval between bowel preparation and the start of colonoscopy was not controlled in this study. As the authors mentioned, this limitation equally affected both groups. However, considering the fact that the time interval is a significant factor affecting the quality of bowel preparation ${ }^{9}$ and proximal colon is frequently involved in poor bowel preparation, we cannot exclude the possibility that the Ottawa bowel preparation scale in this study might have

Correspondence to: Ji Won Kim

Department of Internal Medicine, Seoul National University Boramae Medical Center, Seoul National University College of Medicine, 20 Boramae-ro 5-gil, Dongjak-gu, Seoul 07061, Korea

Tel: +82-2-870-2221, Fax: +82-2-870-3863, E-mail: kjwjor@snu.ac.kr pISSN 1976-2283 eISSN 2005-1212 http://dx.doi.org/10.5009/gnl15659

@) This is an Open Access article distributed under the terms of the Creative Commons Attribution Non-Commercial License (http://creativecommons.org/licenses/by-nc/4.0) which permits unrestricted non-commercial use, distribution, and reproduction in any medium, provided the original work is properly cited. 
been influenced by the time interval between bowel preparation and the start of colonoscopy, particularly in right colon or midcolon. Second, randomization process was not stated precisely in this study. Although the authors mentioned that patients were randomized using random number generation, there were no further descriptions regarding allocation concealment or the time when the randomization process was started. Third, the issue of diet control before bowel preparation needs to be further considered. Moon et al.'s study, ${ }^{7}$ with a study setting similar to Kim et al.'s study ${ }^{8}$ (all split dose for both 4-L PEG and 2-L PEG+Asc groups) indicated no significant difference in the quality of bowel preparation for any of the segments between the two groups. In the study by Moon et al., ${ }^{7}$ patients were limited to a low residue diet for the last 3 days and a liquid diet before 6:00 PM on the day before colonoscopy. However, Kim et al.'s study ${ }^{8}$ allowed regular breakfast and lunch on the day before colonoscopy, which is liberal compared with other studies. $^{7}$ The diet protocol of this study seems very feasible for outpatients because it can reduce its interference in their daily lives. However, the bowel cleansing score in the mid-colon of 2-L $\mathrm{PEG}+$ Asc group was significantly worse than that of 4-L PEG group. Despite some studies supporting liberal diet, ${ }^{10}$ the results of Kim et al.'s study ${ }^{8}$ might suggest 2-L PEG+Asc can have worse bowel preparation efficacy than 4-L PEG in the setting of a less restrictive diet. Further studies need to be conducted on this issue.

Although there are many options for bowel preparation before colonoscopy, we do not have a perfect regimen with completely satisfactory bowel cleansing efficacy, patient preference, compliance, and safety profiles. Kim et al.'s study, ${ }^{8}$ the first to compare acute mucosal inflammation related to 2-L PEG+Asc and 4-L PEG, indicated no significant difference in terms of mucosal injuries between the two groups. In situations when patients are having difficulties in ingesting 4-L PEG which is not tasting good, or when $\mathrm{NaP}$ and Pico are not considered due to the possibility of mucosal injuries particularly in IBD patients or those ingesting NSAIDs, 2-L PEG+Asc can be considered a good alternative to 4-L PEG. Further studies are warranted to develop a more satisfactory regimen with better bowel cleansing efficacy, patient tolerability, and reduced adverse events.

\section{CONFLICTS OF INTEREST}

No potential conflict of interest relevant to this article was reported.

\section{REFERENCES}

1. Lee HS, Byeon JS. Bowel preparation, the first step for a good quality colonoscopy. Intest Res 2014;12:1-2.

2. Froehlich F, Wietlisbach V, Gonvers JJ, Burnand B, Vader JP. Impact of colonic cleansing on quality and diagnostic yield of colonoscopy: the European Panel of Appropriateness of Gastrointestinal Endoscopy European multicenter study. Gastrointest Endosc 2005;61:378-384.

3. Bae SE, Kim KJ, Eum JB, et al. A comparison of $2 \mathrm{~L}$ of polyethylene glycol and $45 \mathrm{~mL}$ of sodium phosphate versus $4 \mathrm{~L}$ of polyethylene glycol for bowel cleansing: a prospective randomized trial. Gut Liver 2013;7:423-429.

4. Ell C, Fischbach W, Keller R, et al. A randomized, blinded, prospective trial to compare the safety and efficacy of three bowelcleansing solutions for colonoscopy (HSG-01*). Endoscopy 2003;35:300-304.

5. Kang MS, Kim TO, Seo EH, et al. Comparison of the efficacy and tolerability between same-day picosulfate and split-dose polyethylene glycol bowel preparation for afternoon colonoscopy: a prospective, randomized, investigator-blinded trial. Intest Res 2014;12:53-59.

6. Lawrance IC, Willert RP, Murray K. Bowel cleansing for colonoscopy: prospective randomized assessment of efficacy and of induced mucosal abnormality with three preparation agents. Endoscopy 2011;43:412-418.

7. Moon CM, Park DI, Choe YG, et al. Randomized trial of 2-L polyethylene glycol + ascorbic acid versus 4-L polyethylene glycol as bowel cleansing for colonoscopy in an optimal setting. J Gastroenterol Hepatol 2014;29:1223-1228.

8. Kim MS, Park J, Park JH, et al. Does polyethylene glycol (PEG) plus ascorbic acid induce more mucosal injuries than split-dose 4-L PEG during bowel preparation? Gut Liver 2016;10:237-243.

9. Kim TK, Kim HW, Kim SJ, et al. Importance of the time interval between bowel preparation and colonoscopy in determining the quality of bowel preparation for full-dose polyethylene glycol preparation. Gut Liver 2014;8:625-631.

10. Aoun E, Abdul-Baki H, Azar C, et al. A randomized single-blind trial of split-dose PEG-electrolyte solution without dietary restriction compared with whole dose PEG-electrolyte solution with dietary restriction for colonoscopy preparation. Gastrointest Endosc 2005;62:213-218. 